\title{
Contribución de la seguridad y salud ocupacional en el desarrollo del sector agroindustrial.
}

"The Contribution of occupational health and safety in the development of the agro-industrial sector".

Ing. Luis Ángel Salazar López Mg. ${ }^{1}$, M.Sc Efraín Velastegui López²

DOI: https://doi.org/10.33262/visionariodigital.v2i3.88

\section{Resumen.}

La investigación realizada se enfoca como principal fundamento el determinar un diagnostico situacional que permita reconocer la situación actual del entorno empresarial de la agroindustria, al mismo tiempo desarrollar médiate la academia y la técnica metodologías que ayuden a obtener la mayor información de las acciones que generan riesgos, accidentes y enfermedades en el puesto de trabajo, que como fin último se otorgue opciones de solución que permitan plantear alternativas para minimizar problemas y generar un ambiente laboral acorde, los procesos de gestión de la seguridad basados en los comportamientos y buenas costumbres de los empresarios del sector, cumpliendo de esta manera con la normativa y evitando perdidas por ausentismo del talento humano. Mediante la investigación exploratoria y descriptiva se conoce la realidad del ecuador en temas de SSO determinando de esta manera que necesidad se llegará a tener como empresas en aspectos éticos y legales, sabiendo que se debe conocer el tema y profundizar métodos que permitan un trabajo digno para el talento humano, y que por ende genere desarrollo en el sector agroindustrial.

Palabras clave: seguridad y salud ocupacional, agroindustria, talento humano, riesgo, accidente, enfermedad laboral, desarrollo empresarial.

\section{Abstract.}

The research carried out is focused as the main basis to determine a situational diagnosis that allows to recognize the current situation of the business environment of the

\footnotetext{
Universidad Técnica de Ambato, Empresa Pública Ambato, Ecuador, la.salazar@uta.edu.ec

2 Universidad Técnica de Ambato, Ambato, Ecuador, le.velastegui@uta.edu.ec 
agroindustry, at the same time develop mediate the academy and technique methodologies that help to obtain the most information of the actions that generate risks , accidents and illnesses in the workplace, that as a final goal is given solution options that allow to propose alternatives to minimize problems and generate a working environment according to the safety management processes based on the behavior and good behavior of businessmen of the sector, thus complying with the regulations and avoiding losses due to absenteeism of human talent. Through exploratory and descriptive research we know the reality of the equator in SSO issues, determining in this way what need to be had as companies in ethical and legal aspects, knowing that the subject must be known and deepening methods that allow decent work for the human talent, and that therefore generates development in the agroindustrial sector.

Keywords: Occupational health and safety, agroindustry, human talent, risk, accident, occupational disease, business development.

\section{Introducción.}

El trabajo es considerado como la fuente del desarrollo del sistema productivo, encaminado en su gran parte por la mano de obra, considerado como una de las trilogías de la economía política los cuales son: TRABAJO, tierra y capital, pilares fundamentales para los principios del progreso de las sociedades, en este contacto la mercancía toma valor en referencia a la producción por su mano de obra, lo cual será la principal base de estudio para el modelo social empresarial (Marx, 1849), en efecto lo que refiere Adam Smith citado por Dobb (2014) el menciona que el trabajo cumple con un proceso productivo que agrega valor a los bienes y servicios, por lo tanto la mano de obra es la inherente en los procesos para el incremento de la permanencia de las organizaciones en el contex to empresarial, debido a la proyección del talento humano Así mismo el trabajo es un asunto social de dignidad y compromiso de la sociedad y las organizaciones productivas que se integran en los sistemas socio económicos de un contexto, por lo cual existen normas de convivencia entre el talento humano y los sectores productivos, que determinen cono objeto el fin común de la justa y equitativa en función de un trabajo digno en todos los aspectos, respetando al ser humano como el principal fomento de la riqueza en las organizaciones (Rodgers, Lee, Swepston, \& Van Daele, 2009), al respecto el equipo investigador determina que la forma de la evolución de las consideraciones del trabajo como proceso productivo a ido cambiando en base al tiempo y demandas de las partes interesadas de una sociedad, por lo cual, el trabajo es esencial para la vida y ayuda al humano a sentirse parte de un segmento importante de la sociedad.

Las exigencias de los ambientes laborales a través del tiempo han generado políticas, normas y controles referente a la prevención de riesgos, accidentes y enfermedades laborales en todo el mundo, ha tomado auge según los índices alarmantes de los riesgos laborales en las organizaciones según la Organización Internacional del Trabajo, por lo cual se ha tornado en un efecto positivo a través debido a que se genera constante mente 
técnicas, procedimientos y reglamentos que se implementa para minimizar los riesgos en el trabajo, en el país según el Plan Nacional para el Buen Vivir en su Objetivo: 9 menciona de manera textual "Garantizar el trabajo digno en todas sus formas", lo cual supone que la supremacía es del trabajo del talento humano sobre el capital en todo su contexto, lo que hace énfasis en que el talento humano es no es un factor de la producción si no un elemento de fomento al desarrollo social (Secretaría Nacional de Planificación y Desarrollo - Senplades, 2013).

En el Ecuador la existen normas que establecen a las instituciones, empresas y empleadores las cuales garanticen a los trabajadores condiciones de seguridad, salud y bienestar en un ambiente de trabajo adecuado que aplaquen para ellos el ejercicio pleno de sus facultades físicas y mentales, a través del fomento del trabajo seguro, la prevención de los accidentes y enfermedades ocupacionales, determinando procesos que disminuyan en un gran porcentaje los riesgos y peligros en los ambientes y condiciones de trabajo.

En el contexto que se desenvuelve la Agroindustria se determina que es uno de los sectores productivos más peligrosos para el manejo del talento humano en base a la cantidad de productos y maquinarias que se utilizan en el proceso productivo "según las estimaciones de la OIT, los trabajadores del mundo sufren 250 millones de accidentes cada año. De un total de 335.000 accidentes laborales mortales anuales, unos 170.000 ocurren en el sector agrícola" (Castañeda \& Gavilán, 2017), por lo cual la presente investigación tendrá como objetivo fundamentar los aspectos legales y técnicos para disminuir los riesgos, accidentes y enfermedades ocupacionales en el trabajo, fundamentado en tres aspectos de suma importancia los cuales son: diagnostico situacional de SSO, Aplicación de las técnicas internacionales de SSO, Aplicación de la normativa en el país.

\section{Marco Teórico Referencial.}

La Organización Internacional del Trabajo (2017) determina que las organizaciones que tienen actividades económicas deben cumplir con normas de seguridad e higiene en el trabajo para contribuir con un ambiente laboral optimo en el puesto laboral, además según estadísticas expuesta por este organismo internacional estima que "2,02 millones de personas mueren cada año a causa de enfermedades y accidentes del trabajo. Otros 317 millones de personas sufren enfermedades relacionadas con el trabajo y cada año se producen unos 337 millones de accidentes laborales mortales y no mortales", de igual manera afecta a un contexto amplio de los indicadores macroeconómicos, sufriendo de esta manera un 4 por ciento del PIB anual mundial, los empleadores asumen costos financieros que podrían llevar a la quiebra de sus organizaciones por no cumplir estas normas.

"Toda persona tendrá derecho a desarrollar sus labores en un ambiente adecuado y propicio, que garantice su salud, integridad, seguridad, higiene y bienestar" artículo 326 
de la Constitución de la República del Ecuador aclara que el trabajo debe ser digno y no presentar ningún indicio de riesgos en el ambiente en el que se desenvuelven los trabajadores, por lo cual es necesario intervenir con metodologías de apoyo conjunto a los empleadores que permitan fomentar trabajos formales y dignos para las sociedad (Asamblea Nacional Constituyente del Ecuador, 2008).

La universalidad de las garantías del trabajo justos, equitativo y digno es un principio rector de la convivencia entre ala industria, estado y sociedad, que permiten entablar mecanismos de desarrollo que permitan interactuar entre modelos de desarrollo en ámbito del mercado laboral, las exigencias de la seguridad social en el país son amplia y recaban necesidades del contexto que permitan proteger a los trabajadores (Congreso Nacional del Ecuador, 2001), por lo tanto, se hace referencia al Reglamento de Seguridad y Salud de los Trabajadores y Mejoramiento del Medio Ambiente de Trabajo en cual de manera textual menciona que la aplicación de " toda actividad laboral y en todo centro de trabajo, teniendo como objetivo la prevención, disminución o eliminación de los riesgos del trabajo y el mejoramiento del medio ambiente de trabajo" (Presidencia de la República del Ecuador, 1986), es importante mencionar que el equipo investigador considera que de manera primordial el tener mayor normativa y mejores estrategia de mitigación de riesgos y enfermedades laborales tendrá como resultado el cumplimiento de las normativas legales y por su consiguiente es necesario intervenir de un proceso de conciencia social para la con las industrias con el fin de entender el contexto del humano sobre el capital, dará mayores resultados y mejor sentido de pertenencia.

Hay sectores en los cuales no se representan cumplimientos de normativas sin que las propuestas de los gobiernos sean de apoyo conciso para las organizaciones productivas es un caso muy referencial la agroindustria que a pasar de los años no deja de ser un contexto olvidado ya que en varias situación existen grandes empresa que por lo general tratan de cumplir con las normas y las MIPYMES que no tienen la suficiente inversión de poder hacerlo, las normas de seguridad y salud ocupación en cualquier ambiro debe ser mediante el apoyo eminente en el área de la problemática social y la intervención de apoyo debe ser inmediata de los organismos que controlan y regulan el sistema social en el trabajo (Conferencia Internacional del Trabajo, 2009), el gobierno debe ser un ente de apoyo y no un sancionador e inquisidor del sector empresarial Agroindustrial.

En referencia del contexto industrial se consideran mecanismos que ayuden a intervenir en los proceso productivos de las empresas por medio del "fomento y la promoción de una cultura de prevención en materia de seguridad y salud son elementos fundamentales para mejorar los resultados relativos a la SST a largo plazo" (Oficina Internacional del Trabajo, 2003), (Conferencia Internacional del Trabajo, 2009).

El Convenio sobre la seguridad y la salud en la agricultura menciona que "los procesos industriales que utilizan productos agrícolas como materia prima, y los servicios 
ISSN 2602-8506

Vol. 2 / No.3/ pág. 24-35. Julio-Septiembre/2018

WwW.visionariodigital.or

conexos", deberán ser estrictamente regulados por los entes de gobierno, es decir debe existir una normativa vigente para la agroindustria la cual no se encuentra desarrollada en el Ecuador (Organización Internacional del Trabajo, 2001).Existen normativas caducas del año 1986 , no se tiene un instrumento que permita a los empresarios de la agroindustria manejar y dirigir de manera consiente la SST por lo cual es necesario proponer por parte de los organismos gubernamentales instructivos pertinentes (Piedra, 2017)

\section{Marco Metodológico.}

El presente trabajo de investigación es cualicuantitativa, la cual se lleva a cabo mediante una investigación descriptiva basado en el método de observación y de investigación documental, que por su contexto necesariamente se deberá hacer un análisis comparativo mediante matrices de riesgos existentes en los medios que permitirán tomar decisiones después de un análisis situacional del sector agroindustrial. Además de ser un tipo de investigación aplicada sustentada desde una investigación básica.

Es necesariamente una investigación una investigación de campo que tiene como instrumentos las encuestas y test pre elaborado por metodologías internacionales aplicadas en otras investigaciones.

El equipo investigador determina que es de suma importancia incluir investigaciones similares que den como resultado un análisis más profundo para determinar el aporte de la SST como desarrollo sustentable y sostenible de la Agroindustria.

En la agroindustria existe varios departamentos que apoyan al proceso productivo de las empresas por lo cual se identificaran de la siguiente manera por medio de una metodología de cadena de valor:

Gráfico1: Cadena de valor.

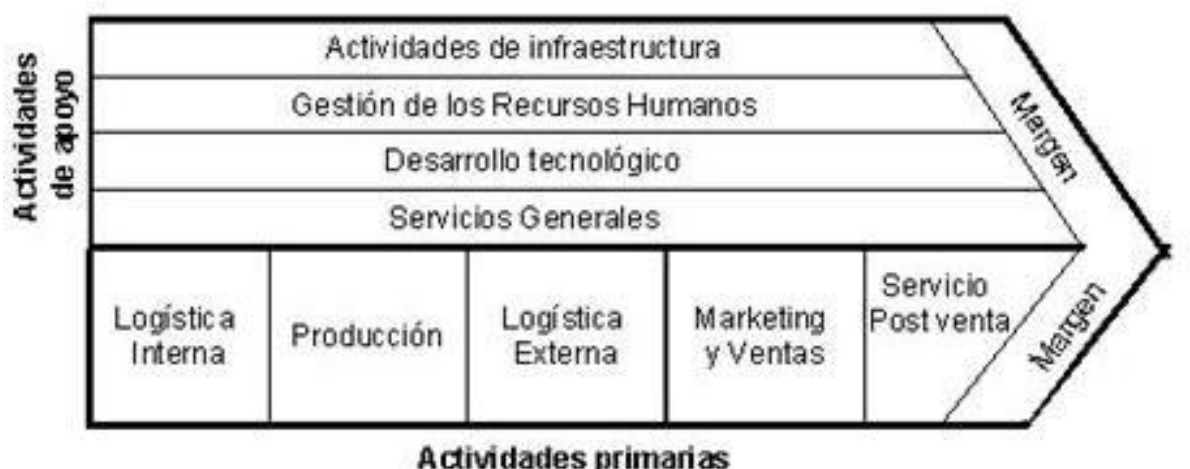

Fuente: (Granda, 2017) 
www.visionariodigital.org

En base al grafico expuesto la investigación se determina desde las actividades primarias ya que, sin las actividades más vulnerables del sistema de Seguridad y Salud en el Trabajo para el sector agroindustrial, una vez ya presentando cual será el enfoque de la investigación de manera subsiguiente se presenta la metodología técnica que se recomienda aplicar en el sector:

Gráfico 2: Matriz de términos.

\begin{tabular}{|l|l|}
\hline Riesgo & Acción y temporización \\
\hline Trivial (T) & No se requiere acción especifica \\
\hline Tolerable (TO) & $\begin{array}{l}\text { No se necesita mejorar la acción preventiva. Sin embargo } \\
\text { se deben considerar soluciones más rentables o mejoras } \\
\text { que no supongan una carga económica importante. } \\
\text { Se requieren comprobaciones periódicas para asegurar } \\
\text { que se mantiene la eficacia de las medidas de control. }\end{array}$ \\
\hline Moderado (M) & $\begin{array}{l}\text { Se deben hacer esfuerzos para reducir el riesgo, } \\
\text { determinando las inversiones precisas. Las medidas para } \\
\text { reducir el riesgo deben implantarse en un perído } \\
\text { determinado. } \\
\text { Cuando el riesgo moderado esta asociado con } \\
\text { consecuencias extremadamente dañinas, se precisará } \\
\text { una acción posterior para establecer, con más precisión, } \\
\text { la probabilidad de daño como base para determinar la } \\
\text { necesidad de mejora de las medidas de control. }\end{array}$ \\
\hline Importante (I) & $\begin{array}{l}\text { No debe comenzarse el trabajo hasta que se haya } \\
\text { reducido el riesgo. Puede que se precisen recursos } \\
\text { considerables para controlar el riesgo. Cuando el riesgo } \\
\text { corresponda a un trabajo que se está realizando, debe } \\
\text { remediarse el problema en un tiempo inferior al de los } \\
\text { riesgos moderados. }\end{array}$ \\
\hline Intolerable (IN) & $\begin{array}{l}\text { No debe comenzar ni continuar el trabajo hasta que se } \\
\text { reduzca el riesgo. Si no es posible reducir el riesgo, } \\
\text { incluso con recursos ilimitados, debe prohibirse el } \\
\text { trabajo. }\end{array}$ \\
\hline
\end{tabular}

Fuente: (Hernández, 2015)

Gráfico 3: Matriz de evaluación de riesgos.

Niveles de riesgo

\begin{tabular}{|c|c|c|c|c|}
\hline & & \multicolumn{3}{|c|}{ Consecuencias } \\
\hline & & $\begin{array}{l}\text { Ligeramente } \\
\text { Dañino } \\
\text { LD }\end{array}$ & $\begin{array}{l}\text { Dañino } \\
\text { D } \\
\end{array}$ & $\begin{array}{l}\text { Extremadamente } \\
\text { Dańino } \\
\text { ED }\end{array}$ \\
\hline \multirow{3}{*}{ Probabilidad } & $\begin{array}{l}\text { Baja } \\
\text { B }\end{array}$ & $\underset{\mathbf{T}}{\text { Riesgo trivial }}$ & $\begin{array}{c}\text { Riesgo tolerable } \\
\text { TO }\end{array}$ & $\begin{array}{l}\text { Riesgo moderado } \\
\text { MO }\end{array}$ \\
\hline & $\begin{array}{l}\text { Media } \\
\text { M }\end{array}$ & $\begin{array}{c}\text { Riesgo tolerable } \\
\text { TO }\end{array}$ & $\begin{array}{l}\text { Riesgo moderado } \\
\text { MO }\end{array}$ & Riesgo importante \\
\hline & $\begin{array}{l}\text { Alta } \\
\text { A }\end{array}$ & $\begin{array}{c}\text { Riesgo moderado } \\
\text { MO }\end{array}$ & Riesgo importante & $\begin{array}{l}\text { Riesgo intolerable } \\
\text { IN }\end{array}$ \\
\hline
\end{tabular}

Fuente: (Hernández, 2015) 
Una vez que se presentan los mecanismos principales para el desarrollo de la presente investigación es necesario considerar la fuente del mecanismo que se toma en base a la investigación de campo, la cual determina de qué manera las agroindustria tienen problemáticas en las reglamentaciones de aplicación para la Seguridad y Salud en el Trabajo.

\section{Análisis de los Resultados.}

El tiempo en el cual un trabajador pasa en una empresa de la agroindustria es necesaria identificarla porque los accidentes y enfermedades laborales se pueden dar según el tiempo en el cual el trabajador se encuentra en la organización, por lo cual los trabajadores de este sector productivo del país tienen una tendencia a ser altamente rotativos, por lo cual un $17 \%$ de trabajadores supera superan la permanencia en una empresa por más de 4 años, esto puede traer problemas debido a que el trabajo arduo que se realiza también puede ser síntoma de desgaste corporal del trabajador que ingrese a una empresa de la agroindustria podría acumular problemas de salud que fueron acontecimientos de empresas que no cumplan con normativas de SST.

Gráfico 4: Tiempo de trabajo en la agroindustria.

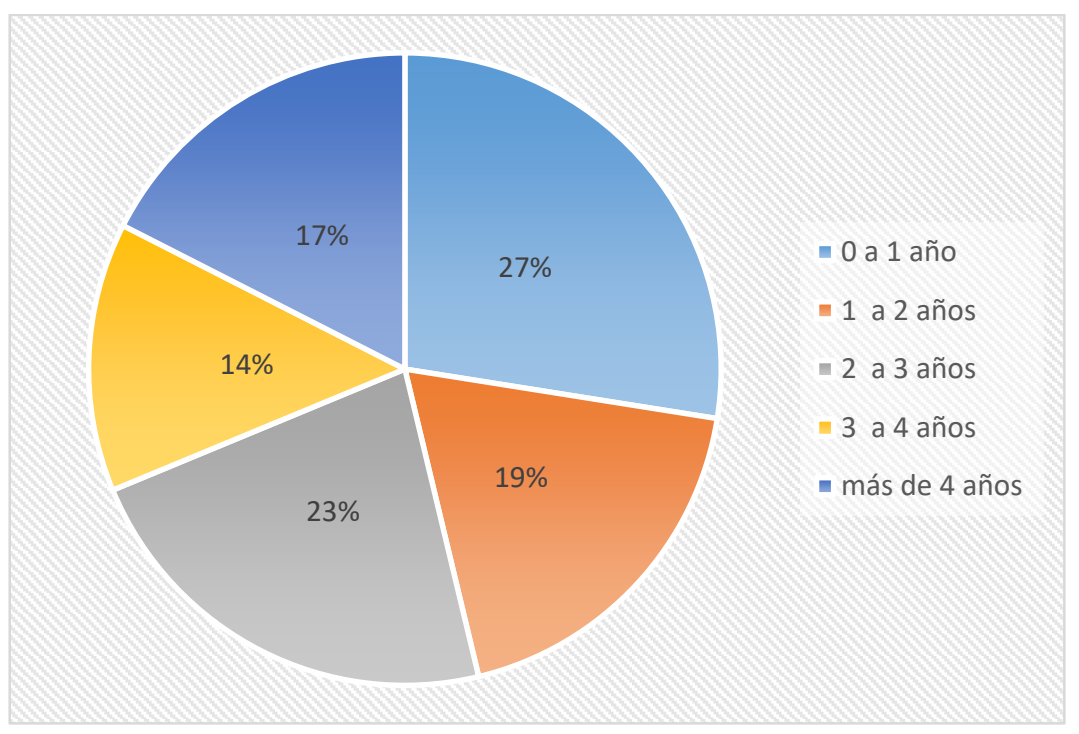

Fuente: Investigación de campo.

El adecuado levantamiento de funciones y procesos en los puestos de trabajo darán como resultado un correcto modelo de identificación de riesgos según las actividades que realicen los trabajadores del sector, de esta manera se mitigan falsos proceso que no agregan valor y podrían ser riesgosos en el trayecto laboral, es preocupante saber que los trabajadores en un $41 \%$ de su población no tienen pleno conocimiento de los correctos 
ISSN 2602-8506

Vol. 2 / No.3/ pág. 24-35. Julio-Septiembre/2018

WWW.visionariodigital.or

procesos y funciones específicas laborales, lo cual podría ser un síntoma focal para riesgos y enfermedades en el trabajo.

Gráfico 5: Cocimiento de las actividades laborales.

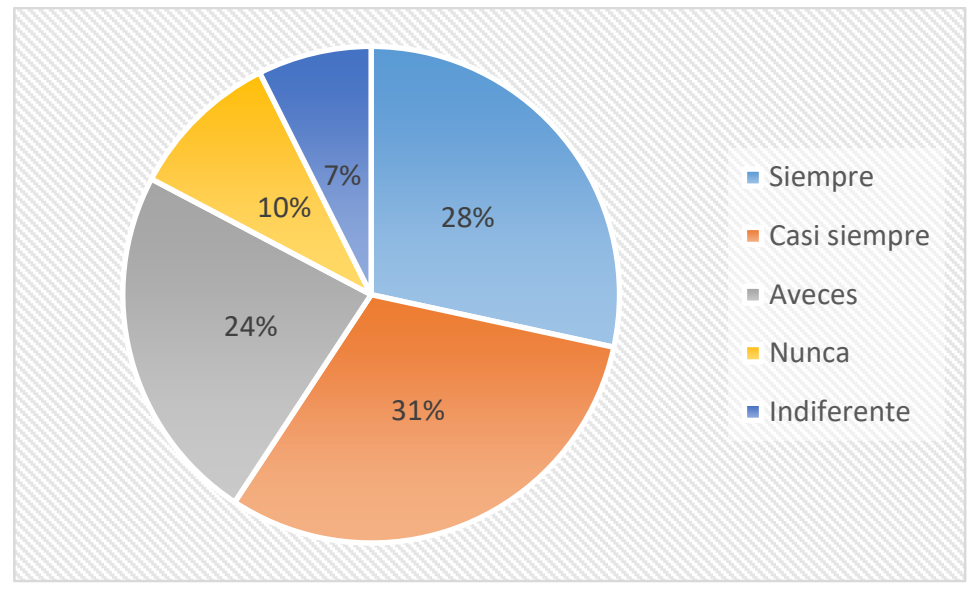

Fuente: Investigación de campo.

Es necesario poner en conocimiento a los trabajadores de aspectos sobre la seguridad y salud en el trabajo, la importancia de salvaguardad su integridad y que eso también será un procesos productivo de la empresa, en la presente investigación solamente el $25 \%$ porcentaje acepta que continuamente se presentan capacitaciones sobre cómo manejar correctamente la mitigación de riesgos laborales, es determinante saber que el $75 \%$ no tienen una formación optima de cómo cuidar sus salud en el trabajo.

Gráfico 6: Subsistema de capacitación de SST.

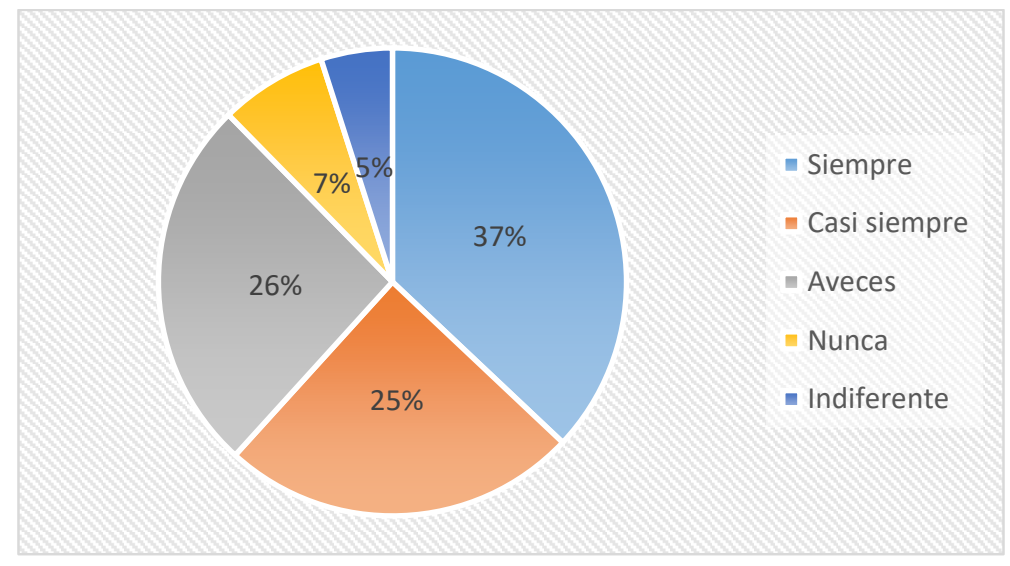

Fuente: Investigación de campo. 
Sabe que los trabajadores han sufrido accidentes laborales de manera continua es imprescindible para tomar decisiones de estos procesos, la mayoría en el transcurso del trabajo y el tiempo de permanencia si han sufrido accidentes laborales en un $37 \%$ de la población trabajadora lo que quiere decir que es un indicador alarmante ya que los riesgos deben ser mínimos y por lo menos restarlos en un $95 \%$ de efectividad.

Gráfico 7: Accidentes laborales en el trabajo.

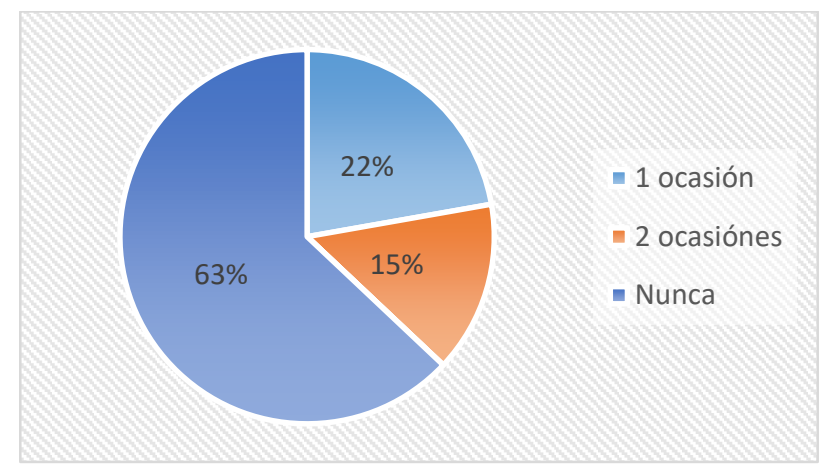

Fuente: Investigación de campo.

Los motivos por los cuales los trabajadores sufren accidentes laborales son múltiples por lo cual hay que realizar la indagación suficiente que permita determinar el motivo exacto o el indicio que permiten tener accidentes laborales en el puesto de trabajo, el $37 \%$ de trabajadores comenten errores por su propia voluntad si respetar los procesos establecidos, sin embargo las limitadas normas de control de SST acompañado del desconocimiento de procedimientos correctos generan un punto focal de alto riesgo siendo este un porcentaje del $49 \%$, en porcentaje siguientes del $14 \%$ y el $12 \%$ son riesgos que se pueden ocasionar por terceras persona y por los equipos de protección y seguridad en el trabajo.

Gráfico 8: Accidentes laborales en el trabajo.

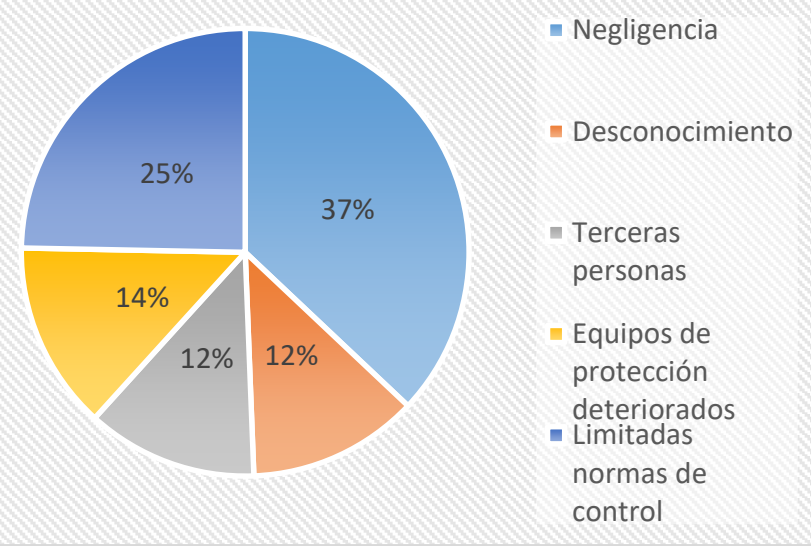

Fuente: Investigación de campo.

Conclusiones. 
- Es importante determinar de manera formal las actividades de los puestos de trabajo, informar y capacitar sobre los procesos específicos que son bases de la producción económica que se vean anclados a plan estratégicos de las empresas.

- Levantan un correcto descriptivo del cargo, delineando de manera minuciosa la cantidad de procesos y cuales son esenciales para que el puesto se encuentre con indicadores positivos, eso mitigará procesos que no son inherentes al entorno laboral, lo cual restará la posibilidad de generar riesgos laborales.

- Al no tener una continuidad laboral en el trabajo y al tener personal rotativo en las empresas de la agroindustria es imprescindible establecer mecanismos de análisis médicos que permitan conocer el estado en el cual ingresa el trabajador, lo cual cuida a las empresas y al contexto productivo de las mismas.

- Capacitar y dar a conocer constante mente las normas de control de lo que se refiere al sistema de seguridad y salud en el trabajo generará conciencia a los colaboradores que se verán obligados a laborar con procedimientos acordes que no pongan en riesgo su integridad física y más a un su vida, al ser el trabajo un derecho constitucional, las empresas deberán prestar todos los mecanismo que pueden generar un trabajo digno y acorde a las funciones de integración social.

- Es necesario el cumplimiento de la ley en temas de Seguridad y Salud en el Trabajo ya que permite no tener problemas con los entes de control, que la inversión que se realiza es mínima en referencia a la cantidad de multas y problemas legales de carácter penal que tendrían los representantes de las empresas de la agroindustria.

\section{Referencias bibliográficas.}

Asamblea Nacional Constituyente del Ecuador . (2008). Monte Cristi: LEXIS.

Castañeda, L., \& Gavilán, G. (2017). Plan de seguridad y salud ocupacional y su efecto en el índice de accidentes de agroindustrial Cayalti S.A. Trujillo: Universidad de Trujillo.

Conferencia Internacional del Trabajo . (2009). Normas de la OIT sobre seguridad y salud en el trabajo . Ginebra: Oficina Internacional del Trabajo Ginebra .

Congreso Nacional del Ecuador. (2001). Ley de Seguridad Social . Quito: LEXIS.

Dobb, M. (2014). Teorías del valor y de la distribución desde Adam Smith: ideología y teoría económica. México D.F.: Siglo XXI.

Granda, C. B. (2017). Gestión técnica del sistema de gestión de seguridad y salud ocupacional en la Dirección Provincial de Orellana del Ministerio de Agricultura Ganadería Acuacultura y Pesca período abril -octubre 2016. Riobamba: Universidad Nacional de Chimborazo. 
Vol. 2 / No.3/ pág. 24-35. Julio-Septiembre/2018

www.visionariodigital.or

Hernández, C. (2015). Incidencia de las actividades del laboratorio de microbioogia ambiental del area agroindustrial de la ESPAM MFL en la seguridad y salud ocupacional de los trabajadores. Calceta: Escuela Superior Politécnica Agropecuaría de Manabí.

Marx, K. (1849). Trabajo asalariado y capital. Berlin: Marxists Internet Archive, 2000.

Oficina Internacional del Trabajo. (2003). Estrategia global en materia de seguridad y salud en el trabajo. Ginebra: Oficina Internacional del Trabajo.

Organización Internacional del Trabajo. (2001). Convenio sobre la seguridad y la salud en la agricultura. Ginebra: Organización Internacional del Trabajo.

Organización Internacional del Trabajo. (06 de 04 de 2017). Organización Internacional del Trabajo. Obtenido de Organización Internacional del Trabajo: http://www.ilo.org/global/standards/subjects-covered-by-international-labourstandards/occupational-safety-and-health/lang--es/index.htm

Piedra, J. P. (31 de Mayo de 2017). Asociación de Exportadores de Banano del Ecuador. Obtenido de Asociación de Exportadores de Banano del Ecuador: http://www.aebe.com.ec/2017/06/seguridad-salud-la-agricultura/

Presidencia de la República del Ecuador. (1986). Reglamento de Seguridad y Salud de los Trabajadores y Mejoramiento del Medio Ambiente de Trabajo. Quito: Presidencia de la República del Ecuador.

Rodgers, G., Lee, E., Swepston, L., \& Van Daele, J. (2009). La Organización Internacional del Trabajo y la lucha por la justicia social, 1919-2009. Ginebra: Organización Internacional del Trabajo.

Secretaría Nacional de Planificación y Desarrollo - Senplades. (2013). Plan Nacional del Buen Vivir. Quito: Senplades.

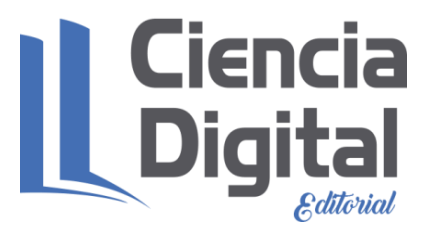


Para citar el artículo indexado.

Salazar L \&Velasteguí E. (2018). Contribución de la seguridad y salud ocupacional en el desarrollo del sector agroindustrial. Revista electrónica Visionario Digital1(1), 24-35. Recuperado desde:

http://cienciadigital.org/revistacienciadigital2/index.php/VisionarioDigital/article/view/88/ $\underline{82}$

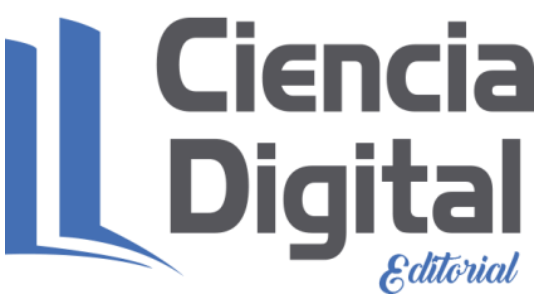

El artículo que se publica es de exclusiva responsabilidad de los autores y no necesariamente reflejan el pensamiento de la Revista Ciencia Digital.

El articulo queda en propiedad de la revista y, por tanto, su publicación parcial y/o total en otro medio tiene que ser autorizado por el director de la Revista Ciencia Digital.
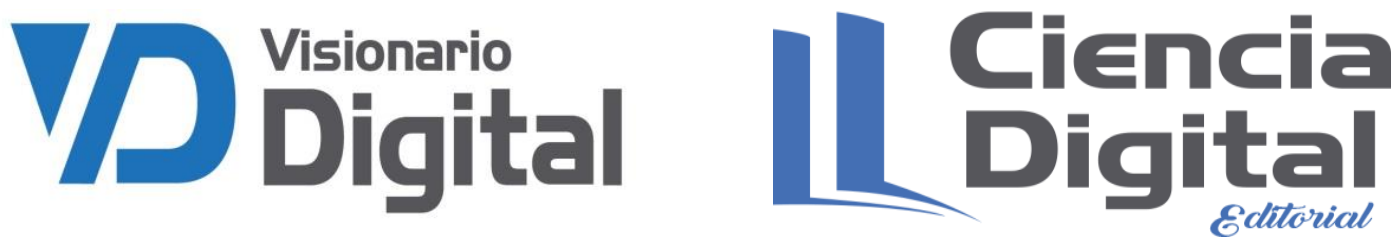